arXiv:0708.3394

DESY $07-130$

MIT-CTP-3857

\title{
General marginal deformations in open superstring field theory
}

\author{
Michael Kiermaier ${ }^{1}$ and Yuji Okawa ${ }^{2}$ \\ 1 Center for Theoretical Physics \\ Massachusetts Institute of Technology \\ Cambridge, MA 02139, USA \\ mkiermai@mit.edu \\ ${ }^{2}$ DESY Theory Group \\ Notkestrasse 85 \\ 22607 Hamburg, Germany \\ yuji.okawa@desy.de
}

\begin{abstract}
We construct analytic solutions of open superstring field theory for any exactly marginal deformation in any boundary superconformal field theory when properly renormalized operator products of the marginal operator are given. Our construction is an extension of the general framework for marginal deformations developed in arXiv:0707.4472 for open bosonic string field theory, and the solutions are based on integrated vertex operators which are closely related to finite deformations in boundary superconformal field theory.
\end{abstract}




\section{Contents}

1 Introduction 1

2 Solutions to the bosonic equation of motion 2

3 Integrated vertex operators in the superstring 5

4 Solutions in the superstring 8

5 Superstring field theory around the deformed background 12

6 Discussion $\quad 14$

6.1 Explicit construction for a class of marginal deformations . . . . . . . . . . . . 14

6.2 More specific assumptions . . . . . . . . . . . . . . . 17

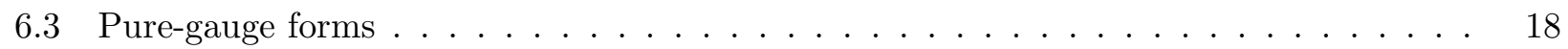

$\begin{array}{ll}\text { A Assumptions } & 20\end{array}$

\section{Introduction}

The purpose of the paper is to extend the general framework for marginal deformations developed in [1] for open bosonic string field theory [2] to open superstring field theory formulated by Berkovits [3] 1 Let us briefly review recent remarkable progress in analytic methods for open string field theory [8]-[31], focusing on marginal deformations. Analytic solutions for marginal deformations were first constructed in [20, 21] for the bosonic string when operator products of the marginal operator are regular, and the solutions were extended to the superstring in $[22,23,25]$. The generalization to marginal deformations with singular operator products was initiated in [21], and solutions to third order in the deformation parameter were constructed. For the special case of the marginal deformation corresponding to the zero mode of the gauge field, solutions to all orders were constructed for the bosonic string in [24] and for the superstring in [28]. The solutions in [24, 28], however, do not satisfy the reality condition on the string field, and a strategy for constructing real solutions was outlined in [28]. See [32]-46] for earlier study of marginal deformations in string field theory and related work.

Analytic solutions for general marginal deformations satisfying the reality condition were recently constructed in [1] for the bosonic string. While previous solutions for marginal deformations in [20, 21, 22, 23, 25] were built from unintegrated vertex operators and $b$-ghost insertions, the solutions in [1] were based on integrated vertex operators which are closely related to finite deformations of boundary conformal field theory (CFT). A change of boundary conditions in boundary CFT can be implemented by properly renormalized exponential operators of an integral of the marginal operator,

\footnotetext{
${ }^{1}$ See [4, 5, 6, 7] for reviews on string field theory.
} 
and a systematic procedure to construct solutions from such renormalized operators was presented in [1]. The general idea of the construction in [1] does not depend on the bosonic nature of the problem, and we expect that the construction can be extended to the superstring. We in fact find that the extension is remarkably simple, and we construct analytic solutions of open superstring field theory to all orders in the deformation parameter satisfying the reality condition.

The organization of the paper is as follows. In section [2 we review the construction in [1] of solutions to the equation of motion for the bosonic string. We use this result later and construct string fields in the superstring satisfying the bosonic equation of motion with the BRST operator in the bosonic theory replaced by the one in the superstring theory. In section 3 we discuss properties of integrated vertex operators in the superstring. In section 4 we construct solutions to the equation of motion of open superstring field theory. This is the main result of the paper. String field theory expanded around the solution was described in [1] using a deformed star product. In section 5 we show that the equation of motion of open superstring field theory expanded around the solution in section 4 can also be described using the deformed star product in [1]. Section 6 is devoted to discussion.

\section{Solutions to the bosonic equation of motion}

The equation of motion of open bosonic string field theory [2] is given by

$$
Q_{B} \Psi+\Psi^{2}=0
$$

where $\Psi$ is the string field of ghost number one and $Q_{B}$ is the BRST operator. Here and in what follows products of string fields are defined by the star product [2]. In this section we review the construction in [1] of solutions to (2.1) for general marginal deformations.

A marginal deformation is generated by a marginal operator $V_{1}(t)$ which is a matter primary field of dimension one. The solutions in [1] are constructed from an operator which implements a change of boundary conditions between two points $a$ and $b$ on the boundary. When operator products of the marginal operator are regular, it is given by

$$
\exp \left[\lambda \int_{a}^{b} d t V_{1}(t)\right]=1+\lambda \int_{a}^{b} d t V_{1}(t)+\frac{\lambda^{2}}{2 !} \int_{a}^{b} d t_{1} \int_{a}^{b} d t_{2} V_{1}\left(t_{1}\right) V_{1}\left(t_{2}\right)+\ldots,
$$

where $\lambda$ is the deformation parameter. When operator products of the marginal operator are singular, we need to renormalize the operator (2.2) properly to make it well defined, and we denote the renormalized operator by

$$
\left[e^{\lambda V(a, b)}\right]_{r},
$$

where

$$
V(a, b) \equiv \int_{a}^{b} d t V_{1}(t)
$$


If the marginal deformation is exactly marginal, there is a one-parameter family of consistent boundary conditions labeled by $\lambda$ and we expect to have a corresponding family of solutions in string field theory. Since the new boundary condition generated by the operator $\left[e^{\lambda V(a, b)}\right]_{r}$ is conformal, the operator $\left[e^{\lambda V(a, b)}\right]_{r}$ should be invariant under the BRST transformation up to additional contributions from the points $a$ and $b$ where the boundary condition changes:

$$
Q_{B} \cdot\left[e^{\lambda V(a, b)}\right]_{r}=\left[e^{\lambda V(a, b)} O_{R}(b)\right]_{r}-\left[O_{L}(a) e^{\lambda V(a, b)}\right]_{r}
$$

Here $O_{L}(a)$ and $O_{R}(b)$ are some local operators at $a$ and $b$, respectively. See the introduction of [1] for more detailed discussion. The solutions in [1] were constructed from the operator $\left[e^{\lambda V(a, b)}\right]_{r}$ as follows. The operator $\left[e^{\lambda V(a, b)}\right]_{r}$ is given in the form of an expansion in $\lambda$ :

$$
\left[e^{\lambda V(a, b)}\right]_{r}=\sum_{n=0}^{\infty} \lambda^{n}\left[V^{(n)}(a, b)\right]_{r}
$$

where

$$
\left[V^{(n)}(a, b)\right]_{r} \equiv \frac{1}{n !}\left[(V(a, b))^{n}\right]_{r} \quad \text { for } \quad n \geq 1 \quad \text { and } \quad\left[V^{(0)}(a, b)\right]_{r} \equiv 1 .
$$

We then define a state $U$ by

$$
U \equiv 1+\sum_{n=1}^{\infty} \lambda^{n} U^{(n)}
$$

where

$$
\left\langle\varphi, U^{(n)}\right\rangle=\left\langle f \circ \varphi(0)\left[V^{(n)}(1, n)\right]_{r}\right\rangle_{\mathcal{W}_{n}} .
$$

Here and in what follows we denote a generic state in the Fock space by $\varphi$ and its corresponding operator in the state-operator mapping by $\varphi(0)$. The conformal transformation $f(\xi)$ is

$$
f(\xi)=\frac{2}{\pi} \arctan \xi
$$

and we denote the conformal transformation of the operator $\varphi(\xi)$ under the map $f(\xi)$ by $f \circ \varphi(\xi)$. The correlation function is evaluated on the surface $\mathcal{W}_{n}$ which is obtained from the upper-half plane of $z$ by the identification $z \sim z+n+1$. We represent $\mathcal{W}_{n}$ in the region where $-1 / 2 \leq \operatorname{Re} z \leq 1 / 2+n$. It follows from (2.5) that the BRST transformation of the operator $\left[V^{(n)}(a, b)\right]_{r}$ takes the form

$$
Q_{B} \cdot\left[V^{(n)}(a, b)\right]_{r}=\sum_{r=1}^{n}\left[V^{(n-r)}(a, b) O_{R}^{(r)}(b)\right]_{r}-\sum_{l=1}^{n}\left[O_{L}^{(l)}(a) V^{(n-l)}(a, b)\right]_{r},
$$

where $O_{L}$ and $O_{R}$ are expanded as follows:

$$
O_{L}=\sum_{n=1}^{\infty} \lambda^{n} O_{L}^{(n)}, \quad O_{R}=\sum_{n=1}^{\infty} \lambda^{n} O_{R}^{(n)} .
$$

Thus the BRST transformation of $U$ can be split into two pieces:

$$
Q_{B} U=A_{R}-A_{L}
$$


with

$$
A_{L}=\sum_{n=1}^{\infty} \lambda^{n} A_{L}^{(n)}, \quad A_{R}=\sum_{n=1}^{\infty} \lambda^{n} A_{R}^{(n)}
$$

where

$$
\begin{aligned}
\left\langle\varphi, A_{L}^{(n)}\right\rangle & =\sum_{l=1}^{n}\left\langle f \circ \varphi(0)\left[O_{L}^{(l)}(1) V^{(n-l)}(1, n)\right]_{r}\right\rangle_{\mathcal{W}_{n}}, \\
\left\langle\varphi, A_{R}^{(n)}\right\rangle & =\sum_{r=1}^{n}\left\langle f \circ \varphi(0)\left[V^{(n-r)}(1, n) O_{R}^{(r)}(n)\right]_{r}\right\rangle_{\mathcal{W}_{n}} .
\end{aligned}
$$

We then define $\Psi_{L}$ by

$$
\Psi_{L} \equiv A_{L} U^{-1}
$$

where $U^{-1}$ is well defined perturbatively in $\lambda$ because $U=1+\mathcal{O}(\lambda)$. The BRST transformation of $\Psi_{L}$ can be calculated as follows:

$$
\begin{aligned}
Q_{B} \Psi_{L} & =Q_{B}\left(A_{L} U^{-1}\right) \\
& =\left(Q_{B} A_{L}\right) U^{-1}+A_{L} U^{-1}\left(Q_{B} U\right) U^{-1} \\
& =\left(Q_{B} A_{L}\right) U^{-1}+A_{L} U^{-1}\left(A_{R}-A_{L}\right) U^{-1} \\
& =\left(Q_{B} A_{L}+A_{L} U^{-1} A_{R}\right) U^{-1}-A_{L} U^{-1} A_{L} U^{-1} \\
& =\left(Q_{B} A_{L}+A_{L} U^{-1} A_{R}\right) U^{-1}-\Psi_{L}^{2} .
\end{aligned}
$$

It was shown in [1] that the relation

$$
Q_{B} A_{L}=-A_{L} U^{-1} A_{R}
$$

holds under a set of assumptions which were argued to be satisfied for any exactly marginal deformation. The equation (2.5) is in fact the first of these assumptions. We list the complete set of assumptions in appendix $\mathrm{A}$. The state $\Psi_{L}$ thus solves the equation of motion:

$$
Q_{B} \Psi_{L}+\Psi_{L}^{2}=0
$$

The solution $\Psi_{L}$, however, does not satisfy the reality condition on the string field, and a solution satisfying the reality condition was generated in [1] from $\Psi_{L}$ by a gauge transformation. The string field $\Psi$ must have a definite parity under the combination of the Hermitean conjugation (hc) and the inverse BPZ conjugation $\left(\mathrm{bpz}^{-1}\right)$ to guarantee that the string field theory action is real [47]. We define the conjugate $X^{\ddagger}$ of a string field $X$ by

$$
X^{\ddagger} \equiv \mathrm{bpz}^{-1} \circ \mathrm{hc}(X) .
$$

The conjugation satisfies

$$
\begin{aligned}
\left(Q_{B} X\right)^{\ddagger} & =-(-1)^{X} Q_{B} X^{\ddagger}, \\
(X Y)^{\ddagger} & =Y^{\ddagger} X^{\ddagger} .
\end{aligned}
$$


Here and in what follows a string field in the exponent of $(-1)$ denotes its Grassmann property: it is 0 mod 2 for a Grassmann-even state and $1 \bmod 2$ for a Grassmann-odd state. In order for $Q_{B} \Psi$ and $\Psi^{2}$ to have the same conjugation property, the Grassmann-odd string field $\Psi$ must satisfy $\Psi^{\ddagger}=\Psi$. This is the reality condition on the string field in open bosonic string field theory. When the renormalized operator $\left[e^{\lambda V(a, b)}\right]_{r}$ preserves the invariance under the reflection where $V_{1}(t)$ is replaced by $V_{1}(a+b-t)$ and when $V_{1}$ is chosen such that the state corresponding to $\lambda V_{1}(0)$ is even under the conjugation 2 we have

$$
U^{\ddagger}=U, \quad\left(U^{-1}\right)^{\ddagger}=U^{-1}, \quad A_{L}^{\ddagger}=A_{R} .
$$

Therefore, a state $\Psi_{R}$ defined by

$$
\Psi_{R} \equiv U^{-1} A_{R}
$$

is the conjugate of $\Psi_{L}$ and solves the equation of motion. The two solutions $\Psi_{L}$ and $\Psi_{R}$ are related by the gauge transformation generated by $U$ :

$$
\Psi_{R}=U^{-1} \Psi_{L} U+U^{-1} Q_{B} U
$$

A solution $\Psi$ satisfying the reality condition is obtained from $\Psi_{L}$ or $\Psi_{R}$ by gauge transformations as follows:

$$
\begin{aligned}
\Psi & =\frac{1}{\sqrt{U}} \Psi_{L} \sqrt{U}+\frac{1}{\sqrt{U}} Q_{B} \sqrt{U} \\
& =\sqrt{U} \Psi_{R} \frac{1}{\sqrt{U}}+\sqrt{U} Q_{B} \frac{1}{\sqrt{U}} \\
& =\frac{1}{2}\left[\frac{1}{\sqrt{U}} \Psi_{L} \sqrt{U}+\sqrt{U} \Psi_{R} \frac{1}{\sqrt{U}}+\frac{1}{\sqrt{U}} Q_{B} \sqrt{U}-\left(Q_{B} \sqrt{U}\right) \frac{1}{\sqrt{U}}\right],
\end{aligned}
$$

where $\sqrt{U}$ and $1 / \sqrt{U}$ are defined perturbatively in $\lambda$. It follows from $(\sqrt{U})^{\ddagger}=\sqrt{U},(1 / \sqrt{U})^{\ddagger}=1 / \sqrt{U}$ and $\Psi_{L}^{\ddagger}=\Psi_{R}$ that the last expression for $\Psi$ in (2.26) manifestly satisfies the reality condition. The three expressions are equivalent because of the relation (2.25).

\section{$3 \quad$ Integrated vertex operators in the superstring}

We expect that integrated vertex operators play a crucial role in extending the construction of solutions in [1] to the superstring. The marginal operator $V_{1}$ in the superstring is the supersymmetry transformation of a superconformal primary field $\widehat{V}_{1 / 2}$ in the matter sector of dimension $1 / 2$ :

$$
V_{1}(t)=G_{-1 / 2} \cdot \widehat{V}_{1 / 2}(t) \equiv \int_{C(t)}\left[\frac{d z}{2 \pi i} T_{F}(z)-\frac{d \bar{z}}{2 \pi i} \widetilde{T}_{F}(\bar{z})\right] \widehat{V}_{1 / 2}(t),
$$

where $T_{F}(z)$ and $\widetilde{T}_{F}(\bar{z})$ are the holomorphic and antiholomorphic components, respectively, of the world-sheet supercurrent, and $C(t)$ is a contour in the upper-half plane which runs from the point $t+\epsilon$

\footnotetext{
${ }^{2}$ If the state corresponding to $V_{1}(0)$ is odd under the conjugation, we set $\lambda=i \tilde{\lambda}$ and take $\tilde{\lambda}$ to be real to satisfy this convention.
} 
on the real axis to the point $t-\epsilon$ on the real axis in the limit $\epsilon \rightarrow 0$ with $\epsilon>0$. An integrated vertex operator in the 0 picture is an integral of $V_{1}$ on the boundary:

$$
V(a, b)=\int_{a}^{b} d t V_{1}(t)=\int_{a}^{b} d t G_{-1 / 2} \cdot \widehat{V}_{1 / 2}(t) .
$$

It is invariant under the BRST transformation up to nonvanishing terms from the end points of the integral region:

$$
\begin{aligned}
Q_{B} \cdot V(a, b) & =\int_{a}^{b} d t \partial_{t}\left[c V_{1}(t)+\eta e^{\phi} \widehat{V}_{1 / 2}(t)\right] \\
& =\left[c V_{1}(b)+\eta e^{\phi} \widehat{V}_{1 / 2}(b)\right]-\left[c V_{1}(a)+\eta e^{\phi} \widehat{V}_{1 / 2}(a)\right] .
\end{aligned}
$$

We use the description of the superconformal ghosts in terms of $\eta, \xi$, and $\phi$ [48, 49], and the BRST operator for the superstring is given by

$$
Q_{B}=\int\left[\frac{d z}{2 \pi i} j_{B}(z)-\frac{d \bar{z}}{2 \pi i} \tilde{\jmath}_{B}(\bar{z})\right]
$$

with

$$
\begin{array}{r}
j_{B}=c T_{B}^{m}+c T_{B}^{\eta \xi}+c T_{B}^{\phi}+\eta e^{\phi} T_{F}^{m}+: b c \partial c:-b \eta \partial \eta e^{2 \phi}, \\
T_{B}^{\eta \xi}=-\eta \partial \xi, \quad T_{B}^{\phi}=-\frac{1}{2} \partial \phi \partial \phi-\partial^{2} \phi
\end{array}
$$

where $T_{B}^{m}$ and $T_{F}^{m}$ are the holomorphic components of the energy-momentum tensor and the supercurrent in the matter sector, respectively, and $\tilde{\jmath}_{B}$ is the antiholomorphic counterpart of $j_{B}$. The operator $V(a, b)$ in the matter sector is obviously annihilated by $\eta_{0}$, which is the zero mode of $\eta$ and plays an important role in open superstring field theory [3]. Since the BRST operator anticommutes with $\eta_{0}$, the operator $Q_{B} \cdot V(a, b)$ is also annihilated by $\eta_{0}$. We can explicitly see that the operator $c V_{1}(t)+\eta e^{\phi} \widehat{V}_{1 / 2}(t)$ which appeared in (3.3) is annihilated by $\eta_{0}$. The operator $c V_{1}(t)+\eta e^{\phi} \widehat{V}_{1 / 2}(t)$ is also annihilated by the BRST operator. This can be seen by acting with $Q_{B}$ on (3.3). In the description of the superconformal ghosts in terms of $\eta, \xi$, and $\phi$ including the sector generated by $\eta_{0}$ and $\xi_{0}$, any BRST-closed operator can be written as an BRST-exact operator because of the existence of a Grassmann-odd operator $R(t)$ satisfying

$$
Q_{B} \cdot R(t)=1
$$

See, for example, footnote 3 of [50]. We choose $R(t)$ to be

$$
R(t) \equiv-c \xi \partial \xi e^{-2 \phi}(t)
$$

Since

$$
\lim _{\epsilon \rightarrow 0} R(t-\epsilon)\left[c V_{1}(t)+\eta e^{\phi} \widehat{V}_{1 / 2}(t)\right]=c \xi e^{-\phi} \widehat{V}_{1 / 2}(t),
$$


we have

$$
c V_{1}(t)+\eta e^{\phi} \widehat{V}_{1 / 2}(t)=Q_{B} \cdot\left[c \xi e^{-\phi} \widehat{V}_{1 / 2}(t)\right] .
$$

Note that the unintegrated vertex operator $c e^{-\phi} \widehat{V}_{1 / 2}$ in the -1 picture with an additional factor of $\xi$ appeared in (3.8). This operator is used in the solution to the linearized equation of motion of open superstring field theory formulated by Berkovits [3], as we will discuss in the next section.

Finite deformations of the boundary CFT are generated by an exponential of $V(a, b)$. When operator products of $V_{1}$ are regular, it is given by $e^{\lambda V(a, b)}$, where $\lambda$ is the deformation parameter. Its BRST transformation is

$$
Q_{B} \cdot e^{\lambda V(a, b)}=\lambda e^{\lambda V(a, b)}\left[c V_{1}(b)+\eta e^{\phi} \widehat{V}_{1 / 2}(b)\right]-\lambda\left[c V_{1}(a)+\eta e^{\phi} \widehat{V}_{1 / 2}(a)\right] e^{\lambda V(a, b)}
$$

if operator products of $\widehat{V}_{1 / 2}$ and an arbitrary number of $V_{1}$ 's are also regular so that (3.3) can be applied even in the presence of further insertions of $V_{1}$ 's. The second term on the right-hand side can be written as

$$
\begin{aligned}
& \lambda\left[c V_{1}(a)+\eta e^{\phi} \widehat{V}_{1 / 2}(a)\right] e^{\lambda V(a, b)} \\
& =\lambda Q_{B} \cdot\left[c \xi e^{-\phi} \widehat{V}_{1 / 2}(a)\right] e^{\lambda V(a, b)} \\
& =\lambda Q_{B} \cdot\left[c \xi e^{-\phi} \widehat{V}_{1 / 2}(a) e^{\lambda V(a, b)}\right]-\lambda^{2}\left[c \xi e^{-\phi} \widehat{V}_{1 / 2}(a)\right] e^{\lambda V(a, b)}\left[c V_{1}(b)+\eta e^{\phi} \widehat{V}_{1 / 2}(b)\right],
\end{aligned}
$$

where we again used the regularity assumption on the operator products. We thus find that

$$
\begin{aligned}
& \lambda Q_{B} \cdot\left[c \xi e^{-\phi} \widehat{V}_{1 / 2}(a) e^{\lambda V(a, b)}\right] \\
& =\lambda\left[c V_{1}(a)+\eta e^{\phi} \widehat{V}_{1 / 2}(a)\right] e^{\lambda V(a, b)}+\lambda^{2}\left[c \xi e^{-\phi} \widehat{V}_{1 / 2}(a)\right] e^{\lambda V(a, b)}\left[c V_{1}(b)+\eta e^{\phi} \widehat{V}_{1 / 2}(b)\right] .
\end{aligned}
$$

This relation, being generalized to the singular case, plays a crucial role in our construction of solutions in open superstring field theory.

When operator products of $V_{1}$ are singular, we need to renormalize the operator $e^{\lambda V(a, b)}$ properly to make it well defined, and we denote the renormalized operator by $\left[e^{\lambda V(a, b)}\right]_{r}$ as before. If the deformation is exactly marginal and preserves superconformal invariance, we assume as in the bosonic case that the BRST transformation of $\left[e^{\lambda V(a, b)}\right]_{r}$ takes the following form:

$$
Q_{B} \cdot\left[e^{\lambda V(a, b)}\right]_{r}=\left[e^{\lambda V(a, b)} O_{R}(b)\right]_{r}-\left[O_{L}(a) e^{\lambda V(a, b)}\right]_{r},
$$

where $O_{L}(a)$ and $O_{R}(b)$ are some Grassmann-odd local operators at $a$ and $b$, respectively. The operators $\left[O_{L}(a) e^{\lambda V(a, b)}\right]_{r}$ and $\left[e^{\lambda V(a, b)} O_{R}(b)\right]_{r}$ are annihilated by $\eta_{0}$, as we discussed before. To leading order in $\lambda$ they are determined from (3.3) and given by

$$
\begin{aligned}
& {\left[O_{L}(a) e^{\lambda V(a, b)}\right]_{r}=\lambda\left[c V_{1}(a)+\eta e^{\phi} \widehat{V}_{1 / 2}(a)\right]+\mathcal{O}\left(\lambda^{2}\right),} \\
& {\left[e^{\lambda V(a, b)} O_{R}(b)\right]_{r}=\lambda\left[c V_{1}(b)+\eta e^{\phi} \widehat{V}_{1 / 2}(b)\right]+\mathcal{O}\left(\lambda^{2}\right) .}
\end{aligned}
$$

In the regular case, we find from the exact expression in (3.10) that

$$
O_{L}^{\text {regular }}=O_{R}^{\text {regular }}=\lambda\left[c V_{1}+\eta e^{\phi} \widehat{V}_{1 / 2}\right],
$$


and there are no higher-order corrections to the operators $O_{L}$ and $O_{R}$.

Let us introduce the following operators:

$$
\begin{aligned}
& {\left[\widehat{O}_{L}(a) e^{\lambda V(a, b)}\right]_{r} \equiv \lim _{\epsilon \rightarrow 0} R(a-\epsilon)\left[O_{L}(a) e^{\lambda V(a, b)}\right]_{r},} \\
& {\left[e^{\lambda V(a, b)} \widehat{O}_{R}(b)\right]_{r} \equiv-\lim _{\epsilon \rightarrow 0}\left[e^{\lambda V(a, b)} O_{R}(b)\right]_{r} R(b+\epsilon) .}
\end{aligned}
$$

These are generalizations of $\lambda c \xi e^{-\phi} \widehat{V}_{1 / 2}(a) e^{\lambda V(a, b)}$ and $\lambda e^{\lambda V(a, b)} c \xi e^{-\phi} \widehat{V}_{1 / 2}(b)$ in the regular case. The ghost sector couples to the matter sector only through $c$ and $\eta e^{\phi}$ in the BRST current, and the operator products of $c \xi \partial \xi e^{-2 \phi}$ with $c, \eta e^{\phi}$, and their derivatives are regular. The limit $\epsilon \rightarrow 0$ in (3.16) is therefore regular. To leading order in $\lambda$ these operators reduce to

$$
\begin{aligned}
{\left[\widehat{O}_{L}(a) e^{\lambda V(a, b)}\right]_{r} } & =\lambda c \xi e^{-\phi} \widehat{V}_{1 / 2}(a)+\mathcal{O}\left(\lambda^{2}\right), \\
{\left[e^{\lambda V(a, b)} \widehat{O}_{R}(b)\right]_{r} } & =\lambda c \xi e^{-\phi} \widehat{V}_{1 / 2}(b)+\mathcal{O}\left(\lambda^{2}\right) .
\end{aligned}
$$

The BRST transformation of $\left[\widehat{O}_{L}(a) e^{\lambda V(a, b)}\right]_{r}$ can be calculated from (3.6) and from the BRST transformation of $\left[O_{L}(a) e^{\lambda V(a, b)}\right]_{r}$. When the deformation is exactly marginal and preserves superconformal invariance, we assume that the BRST transformation of $\left[O_{L}(a) e^{\lambda V(a, b)}\right]_{r}$ is given by

$$
Q_{B} \cdot\left[O_{L}(a) e^{\lambda V(a, b)}\right]_{r}=-\left[O_{L}(a) e^{\lambda V(a, b)} O_{R}(b)\right]_{r}
$$

See the introduction of [1] for more detailed discussion. The BRST transformation of $\left[\widehat{O}_{L}(a) e^{\lambda V(a, b)}\right]_{r}$ is then given by

$$
\begin{aligned}
Q_{B} \cdot\left[\widehat{O}_{L}(a) e^{\lambda V(a, b)}\right]_{r} & =Q_{B} \cdot\left[\lim _{\epsilon \rightarrow 0} R(a-\epsilon)\left[O_{L}(a) e^{\lambda V(a, b)}\right]_{r}\right] \\
& =\left[O_{L}(a) e^{\lambda V(a, b)}\right]_{r}+\lim _{\epsilon \rightarrow 0} R(a-\epsilon)\left[O_{L}(a) e^{\lambda V(a, b)} O_{R}(b)\right]_{r}
\end{aligned}
$$

This is a generalization of (3.12). Similarly, we find

$$
\begin{aligned}
Q_{B} \cdot\left[e^{\lambda V(a, b)} \widehat{O}_{R}(b)\right]_{r} & =-Q_{B} \cdot\left[\lim _{\epsilon \rightarrow 0}\left[e^{\lambda V(a, b)} O_{R}(b)\right]_{r} R(b+\epsilon)\right] \\
& =\left[e^{\lambda V(a, b)} O_{R}(b)\right]_{r}+\lim _{\epsilon \rightarrow 0}\left[O_{L}(a) e^{\lambda V(a, b)} O_{R}(b)\right]_{r} R(b+\epsilon) .
\end{aligned}
$$

As we mentioned before, the relations (3.19) and (3.20) play a crucial role in the construction of the general superstring solutions in the next section.

\section{Solutions in the superstring}

In this section we construct solutions for general marginal deformations in open superstring field theory formulated by Berkovits [3]. The equation of motion is

$$
\eta_{0}\left(e^{-\Phi} Q_{B} e^{\Phi}\right)=0,
$$


where $\Phi$ is the superstring field of ghost number zero. The leading term in the expansion of (4.1) in $\Phi$ is given by

$$
Q_{B} \eta_{0} \Phi+\mathcal{O}\left(\Phi^{2}\right)=0
$$

where we have used $\left\{Q_{B}, \eta_{0}\right\}=0$. To leading order in the deformation parameter $\lambda$, a solution $\Phi$ associated with an exactly marginal deformation takes the form

$$
\langle\varphi, \Phi\rangle=\lambda\left\langle f \circ \varphi(0) c \xi e^{-\phi} \widehat{V}_{1 / 2}(1)\right\rangle_{\mathcal{W}_{1}}+\mathcal{O}\left(\lambda^{2}\right),
$$

where $\widehat{V}_{1 / 2}$ is the superconformal primary operator corresponding to the marginal deformation, as introduced in section 3. The term of (4.3) at $\mathcal{O}(\lambda)$ solves the equation of motion to linear order in $\Phi$ given in (4.2) because $\eta_{0}$ eliminates the operator $\xi$ and the remaining unintegrated vertex operator $c e^{-\phi} \widehat{V}_{1 / 2}$ in the -1 picture is annihilated by the BRST operator.

In 22] Erler proposed to solve the following equation:

$$
e^{-\Phi} Q_{B} e^{\Phi}=\Psi
$$

where $\Psi$ satisfies

$$
Q_{B} \Psi+\Psi^{2}=0, \quad \eta_{0} \Psi=0,
$$

and to linear order in $\lambda$ the state $\Psi$ reduces to

$$
\langle\varphi, \Psi\rangle=\lambda\left\langle f \circ \varphi(0) Q_{B} \cdot\left[c \xi e^{-\phi} \widehat{V}_{1 / 2}(1)\right]\right\rangle_{\mathcal{W}_{1}}+\mathcal{O}\left(\lambda^{2}\right)
$$

Namely, the state $\Psi$ is a pure-gauge string field with respect to the gauge transformation of bosonic string field theory, while we use the BRST operator of the superstring. Since the left-hand side of (4.4) also takes a pure-gauge form, we expect a solution $\Phi$ to the equation (4.4) of the form (4.3). Since $\Psi$ is annihilated by $\eta_{0}$, the solution of (4.4) also solves the equation of motion (4.1).

In [22] such a pure-gauge string field $\Psi$ was constructed from the solution of open bosonic string field theory in [20, 21] by replacing the unintegrated vertex operator $c V_{1}$ in the bosonic string with $Q_{B} \cdot\left[c \xi e^{-\phi} \widehat{V}_{1 / 2}\right]$ in the superstring. Then the equation (4.4) was solved and solutions for marginal deformations were constructed in open superstring field theory when operator products of the marginal operator are regular [22, 25].

We can also obtain pure-gauge string fields satisfying (4.5) using the construction of solutions for bosonic string field theory in [1], which covers the case where operator products of the marginal operator are singular. As we reviewed in section 2, the solutions in [1] are constructed from the operator $\left[e^{\lambda V(a, b)}\right]_{r}$ under the assumptions listed in appendix A. String fields in the superstring satisfying (4.5) can be constructed from the operator $\left[e^{\lambda V(a, b)}\right]_{r}$ with $V_{1}=G_{-1 / 2} \cdot \widehat{V}_{1 / 2}$ as introduced in (3.1) of section 3 because all the assumptions listed in appendix A are expected to be satisfied when the deformation corresponding to $\widehat{V}_{1 / 2}$ is exactly marginal and preserves superconformal invariance. All the solutions in section 2 have the same leading term in $\lambda$ given by $\lambda A_{L}^{(1)}+\mathcal{O}\left(\lambda^{2}\right)$, where $A_{L}^{(n)}$ 
is defined in (2.15) 3 As $A_{L}^{(1)}$ is determined by the leading term of $\left[O_{L}(a) e^{\lambda V(a, b)}\right]_{r}$ which is given in (3.14) for the superstring case, we find

$$
\begin{aligned}
\left\langle\varphi, A_{L}^{(1)}\right\rangle & =\left\langle f \circ \varphi(0) O_{L}^{(1)}(1)\right\rangle_{\mathcal{W}_{1}} \\
& =\left\langle f \circ \varphi(0)\left[c V_{1}(1)+\eta e^{\phi} \widehat{V}_{1 / 2}(1)\right]\right\rangle_{\mathcal{W}_{1}} \\
& =\left\langle f \circ \varphi(0) Q_{B} \cdot\left[c \xi e^{-\phi} \widehat{V}_{1 / 2}(1)\right]\right\rangle_{\mathcal{W}_{1}}
\end{aligned}
$$

where we have used (3.9). Therefore, the condition (4.6) is satisfied. The solutions in [1] are built from the operator $\left[e^{\lambda V(a, b)}\right]_{r}$ and its BRST transformation which are both annihilated by $\eta_{0}$, and thus the second condition in (4.5) is also satisfied. We can thus construct superstring solutions for marginal deformations from the bosonic solutions of [1] by solving (4.4).

The leading term of the superstring solution $\Phi$ in (4.3) is built from the leading term of the operator $\left[\widehat{O}_{L}(a) e^{\lambda V(a, b)}\right]_{r}$, as can be seen from (3.17). We therefore expect that the operator $\left[\widehat{O}_{L}(a) e^{\lambda V(a, b)}\right]_{r}$ plays an important role in the construction of superstring solutions. Just as $A_{L}$ and $A_{R}$ are constructed from the operators $\left[O_{L}(1) e^{\lambda V(1, n)}\right]_{r}$ and $\left[e^{\lambda V(1, n)} O_{R}(n)\right]_{r}$ at $\mathcal{O}\left(\lambda^{n}\right)$, respectively, we introduce states $\widehat{A}_{L}$ and $\widehat{A}_{R}$ which are constructed from $\left[\widehat{O}_{L}(1) e^{\lambda V(1, n)}\right]_{r}$ and $\left[e^{\lambda V(1, n)} \widehat{O}_{R}(n)\right]_{r}$ at $\mathcal{O}\left(\lambda^{n}\right)$. We define

$$
\widehat{A}_{L}=\sum_{n=1}^{\infty} \lambda^{n} \widehat{A}_{L}^{(n)}, \quad \widehat{A}_{R}=\sum_{n=1}^{\infty} \lambda^{n} \widehat{A}_{R}^{(n)}
$$

with

$$
\begin{aligned}
\left\langle\varphi, \widehat{A}_{L}^{(n)}\right\rangle & =\lim _{\epsilon \rightarrow 0} \sum_{l=1}^{n}\left\langle f \circ \varphi(0) R(1-\epsilon)\left[O_{L}^{(l)}(1) V^{(n-l)}(1, n)\right]_{r}\right\rangle_{\mathcal{W}_{n}}, \\
\left\langle\varphi, \widehat{A}_{R}^{(n)}\right\rangle & =-\lim _{\epsilon \rightarrow 0} \sum_{r=1}^{n}\left\langle f \circ \varphi(0)\left[V^{(n-r)}(1, n) O_{R}^{(r)}(n)\right]_{r} R(n+\epsilon)\right\rangle_{\mathcal{W}_{n}} .
\end{aligned}
$$

The states $\widehat{A}_{L}$ and $\widehat{A}_{R}$ are related by the conjugation:

$$
\left(\widehat{A}_{L}\right)^{\ddagger}=-\widehat{A}_{R}
$$

This can be shown as follows. The state $R$ corresponding to the operator $R(0)$ satisfies $Q_{B} R=|0\rangle$ and thus $R^{\ddagger}=R$, which follows from $|0\rangle^{\ddagger}=|0\rangle$ and (2.21). Following the argument in $\S 2.2 .1$ of [1], the operator $R(1-\epsilon)$ on $\mathcal{W}_{n}$ in the definition of $\widehat{A}_{L}^{(n)}$ is mapped to $R(n+\epsilon)$ under the conjugation. The relation (4.10) then follows from $A_{L}^{\ddagger}=A_{R}$. The BRST transformations of $\widehat{A}_{L}$ and $\widehat{A}_{R}$ can be derived from those of $A_{L}$ and $A_{R}$. The BRST transformation of $A_{L}$ is presented in (2.18), and using (2.13) we find $Q_{B} A_{R}=Q_{B}\left(Q_{B} U+A_{L}\right)=Q_{B} A_{L}$. Thus we have

$$
Q_{B} A_{L}=-A_{L} U^{-1} A_{R}, \quad Q_{B} A_{R}=-A_{L} U^{-1} A_{R}
$$

\footnotetext{
${ }^{3}$ The leading terms of the bosonic solutions $\Psi_{L}, \Psi_{R}$, and $\Psi$ are $\lambda A_{L}^{(1)}, \lambda A_{R}^{(1)}$, and $\lambda\left(A_{L}^{(1)}+A_{R}^{(1)}\right) / 2$, respectively. Because $O_{R}^{(1)}=O_{L}^{(1)}$, we have $A_{R}^{(1)}=A_{L}^{(1)}$ and thus all three solutions are equivalent to leading order.
} 
Using the identities (3.19) and (3.20), the BRST transformations of $\widehat{A}_{L}$ and $\widehat{A}_{R}$ are given by

$$
Q_{B} \widehat{A}_{L}=A_{L}+\widehat{A}_{L} U^{-1} A_{R}, \quad Q_{B} \widehat{A}_{R}=A_{R}-A_{L} U^{-1} \widehat{A}_{R}
$$

These relations hold when the assumptions in appendix $\mathrm{A}$ are satisfied.

We now claim that $\Phi_{L}$ and $\Phi_{R}$ defined by

$$
e^{\Phi_{L}}=1+\widehat{A}_{L} U^{-1}, \quad e^{-\Phi_{R}}=1-U^{-1} \widehat{A}_{R}
$$

solve the equation (4.4) with $\Psi$ being $\Psi_{L}=A_{L} U^{-1}$ and $\Psi_{R}=U^{-1} A_{R}$, respectively, defined in section 2, Using the relations (2.13) and (4.12), we have

$$
\begin{aligned}
Q_{B} e^{\Phi_{L}} & =\left(A_{L}+\widehat{A}_{L} U^{-1} A_{R}\right) U^{-1}-\widehat{A}_{L} U^{-1}\left(A_{R}-A_{L}\right) U^{-1} \\
& =\left(1+\widehat{A}_{L} U^{-1}\right) A_{L} U^{-1} \\
& =e^{\Phi_{L}} \Psi_{L}
\end{aligned}
$$

and

$$
\begin{aligned}
Q_{B} e^{-\Phi_{R}} & =-U^{-1}\left(A_{R}-A_{L} U^{-1} \widehat{A}_{R}\right)+U^{-1}\left(A_{R}-A_{L}\right) U^{-1} \widehat{A}_{R} \\
& =-U^{-1} A_{R}\left(1-U^{-1} \widehat{A}_{R}\right) \\
& =-\Psi_{R} e^{-\Phi_{R}} .
\end{aligned}
$$

Therefore,

$$
e^{-\Phi_{L}} Q_{B} e^{\Phi_{L}}=\Psi_{L}, \quad e^{-\Phi_{R}} Q_{B} e^{\Phi_{R}}=-\left(Q_{B} e^{-\Phi_{R}}\right) e^{\Phi_{R}}=\Psi_{R} .
$$

Since $\Psi_{L}$ and $\Psi_{R}$ are annihilated by $\eta_{0}$, the states $\Phi_{L}$ and $\Phi_{R}$ solve the equation of motion (4.1).

The reality condition on the superstring field $\Phi$ is $\Phi^{\ddagger}=-\Phi$, or

$$
\left(e^{\Phi}\right)^{\ddagger}=e^{-\Phi} .
$$

The solutions $\Phi_{L}$ and $\Phi_{R}$ do not satisfy the reality condition. In fact, we find

$$
\left(e^{\Phi_{L}}\right)^{\ddagger}=e^{-\Phi_{R}},
$$

which follows directly from (4.10) and the definitions (4.13). However, we can generate a real solution from $\Phi_{L}$ and $\Phi_{R}$ by generalizing the method in appendix B of [22]. We claim that $\Phi$ defined by

$$
e^{\Phi}=\left(\sqrt{e^{\Phi_{L}} U e^{-\Phi_{R}}}\right)^{-1}\left(e^{\Phi_{L}} \sqrt{U}\right)
$$

satisfies the reality condition and solves the equation of motion. The state $\Phi$ is well defined to all orders in $\lambda$ because $e^{\Phi_{L}} U e^{-\Phi_{R}}=1+\mathcal{O}(\lambda)$ and $U=1+\mathcal{O}(\lambda)$. Using the relations

$$
e^{\Phi_{L}} U e^{-\Phi_{R}}=\left(e^{\Phi_{L}} \sqrt{U}\right)\left(e^{\Phi_{L}} \sqrt{U}\right)^{\ddagger}, \quad\left(e^{\Phi_{L}} U e^{-\Phi_{R}}\right)^{\ddagger}=e^{\Phi_{L}} U e^{-\Phi_{R}},
$$


we have

$$
\begin{aligned}
\left(e^{\Phi}\right)^{\ddagger} e^{\Phi} & =\left(e^{\Phi_{L}} \sqrt{U}\right)^{\ddagger}\left(\sqrt{\left(e^{\Phi_{L}} \sqrt{U}\right)\left(e^{\Phi_{L}} \sqrt{U}\right)^{\ddagger}}\right)^{-1}\left(\sqrt{\left(e^{\Phi_{L} \sqrt{U}}\right)\left(e^{\Phi_{L} \sqrt{U}}\right)^{\ddagger}}\right)^{-1}\left(e^{\Phi_{L}} \sqrt{U}\right) \\
& =\left(e^{\Phi_{L}} \sqrt{U}\right)^{\ddagger}\left(\left(e^{\Phi_{L}} \sqrt{U}\right)\left(e^{\Phi_{L}} \sqrt{U}\right)^{\ddagger}\right)^{-1}\left(e^{\Phi_{L}} \sqrt{U}\right) \\
& =1 .
\end{aligned}
$$

Therefore, $\left(e^{\Phi}\right)^{\ddagger}=e^{-\Phi}$ and the reality condition (4.17) is satisfied.

The state $\Phi$ defined in (4.19) is related to the solution $\Phi_{L}$ in the following way:

$$
e^{\Phi}=\Omega e^{\Phi_{L}} \Lambda
$$

where $\Omega=\left(\sqrt{e^{\Phi_{L}} U e^{-\Phi_{R}}}\right)^{-1}$ and $\Lambda=\sqrt{U}$. If $\Omega$ is annihilated by $Q_{B}$ and $\Lambda$ is annihilated by $\eta_{0}$, the state $\Phi$ is a gauge transformation of $\Phi_{L}$ and thus satisfies the equation of motion. It is obvious that the state $\sqrt{U}$ is annihilated by $\eta_{0}$. The state $\left(\sqrt{e^{\Phi_{L} U} e^{-\Phi_{R}}}\right)^{-1}$ is annihilated by $Q_{B}$ if $e^{\Phi_{L}} U e^{-\Phi_{R}}$ is annihilated by $Q_{B}$. It can be shown as follows:

$$
\begin{aligned}
Q_{B}\left(e^{\Phi_{L}} U e^{-\Phi_{R}}\right) & =e^{\Phi_{L}}\left(\Psi_{L} U+Q_{B} U-U \Psi_{R}\right) e^{-\Phi_{R}} \\
& =e^{\Phi_{L}}\left(A_{L}+Q_{B} U-A_{R}\right) e^{-\Phi_{R}} \\
& =0
\end{aligned}
$$

where we used (2.13) in the last step. This completes the construction of real solutions in open superstring field theory for general marginal deformations under the assumptions listed in appendix A. Incidentally, the solution $\Phi$ satisfies the equation (4.4) with the real solution $\Psi$ of [1] given in (2.26):

$$
\begin{aligned}
Q_{B} e^{\Phi} & =\left(\sqrt{e^{\Phi_{L} U e^{-\Phi_{R}}}}\right)^{-1} Q_{B}\left(e^{\Phi_{L}} \sqrt{U}\right) \\
& =\left(\sqrt{e^{\Phi_{L} U e^{-\Phi_{R}}}}\right)^{-1} e^{\Phi_{L}}\left(\Psi_{L} \sqrt{U}+Q_{B} \sqrt{U}\right) \\
& =\left(\sqrt{e^{\Phi_{L} U e^{-\Phi_{R}}}}\right)^{-1} e^{\Phi_{L}} \sqrt{U}\left(\frac{1}{\sqrt{U}} \Psi_{L} \sqrt{U}+\frac{1}{\sqrt{U}} Q_{B} \sqrt{U}\right) \\
& =e^{\Phi} \Psi .
\end{aligned}
$$

Since $\Psi$ is annihilated by $\eta_{0}$, we have reconfirmed that $\Phi$ solves the equation of motion (4.1).

\section{$5 \quad$ Superstring field theory around the deformed background}

It was shown in [1] that the action of open bosonic string field theory expanded around the real solution (2.26) can be written in terms of deformed algebraic structures defined by

$$
\begin{aligned}
X \star Y & \equiv X U^{-1} Y, \\
\mathcal{Q} X & \equiv Q_{B} X+A_{L} \star X-(-1)^{X} X \star A_{R}=Q_{B} X+\Psi_{L} X-(-1)^{X} X \Psi_{R}, \\
\langle\langle X, Y\rangle\rangle & \equiv\left\langle X, U^{-1} Y U^{-1}\right\rangle
\end{aligned}
$$


for arbitrary string fields $X$ and $Y$. When $\lambda=0$, the deformed structures reduce to their undeformed counterparts because $U=1+\mathcal{O}(\lambda)$ and $A_{L}, A_{R}, \Psi_{L}$, and $\Psi_{R}$ are of $\mathcal{O}(\lambda)$. The equation of motion derived from the action in terms of the deformed structures is

$$
\mathcal{Q} \delta \Psi+\delta \Psi \star \delta \Psi=0
$$

where $\delta \Psi$ is related to the original string field $\Psi$ expanded around the real solution (2.26), which we now denote by $\Psi_{0}$, as follows:

$$
\Psi=\Psi_{0}+\frac{1}{\sqrt{U}} \delta \Psi \frac{1}{\sqrt{U}}
$$

The deformed structures obey the expected algebraic relations

$$
\begin{aligned}
\mathcal{Q}^{2} X & =0, \\
\mathcal{Q}(X \star Y) & =(\mathcal{Q} X) \star Y+(-1)^{X} X \star(\mathcal{Q} Y), \\
\langle\langle X, Y\rangle\rangle & =(-1)^{X Y}\langle\langle Y, X\rangle\rangle, \\
\langle\langle\mathcal{Q} X, Y\rangle\rangle & =-(-1)^{X}\langle\langle X, \mathcal{Q} Y\rangle\rangle, \\
\langle\langle X, Y \star Z\rangle\rangle & =\langle\langle X \star Y, Z\rangle\rangle,
\end{aligned}
$$

which are necessary for a consistent formulation of string field theory. Since open superstring field theory [3] is formulated using the algebraic structures of open bosonic string field theory, the action of open superstring field theory written in terms of the deformed structures is consistent. It is expected to describe fluctuations around the background corresponding to the solution (4.19) in terms of a redefined string field $\delta \Phi$. We show in this section that this is indeed the case and derive the relation between $\delta \Phi$ and the original string field $\Phi$ analogous to the relation (5.3) between $\Psi$ and $\delta \Psi$ for the bosonic case found in [1].

To formulate superstring field theory using the deformed algebraic structures, we first have to introduce an exponential operator $\exp _{\star}[X]$ of the deformed star algebra. As can be seen from the definition (5.1), the state $U$ plays the role of the identity element of the deformed star algebra:

$$
X \star U=U \star X=X .
$$

We thus define $\exp _{\star}[X]$ by

$$
\exp _{\star}[X]=U+X+\frac{1}{2 !} X \star X+\frac{1}{3 !} X \star X \star X+\ldots=U+\sum_{n=1}^{\infty} \frac{1}{n !} \underbrace{X \star X \star \ldots \star X}_{n \text { times }} .
$$

The equation of motion derived from the action using the deformed algebraic structures is

$$
\eta_{0}\left(\exp _{\star}[-\delta \Phi] \star \mathcal{Q} \exp _{\star}[\delta \Phi]\right)=0
$$

To determine the relation between $\delta \Phi$ and the original string field $\Phi$, let us express (5.7) in terms of the undeformed star product and the BRST operator $Q_{B}$. The exponential operator $\exp _{\star}[X]$ can be written as

$$
\exp _{\star}[X]=\sqrt{U} e^{\frac{1}{\sqrt{U}} X \frac{1}{\sqrt{U}} \sqrt{U}} .
$$


We thus find

$$
\begin{aligned}
& \frac{1}{\sqrt{U}}\left[\eta_{0}\left(\exp _{\star}[-\delta \Phi] \star \mathcal{Q} \exp _{\star}[\delta \Phi]\right)\right] \frac{1}{\sqrt{U}} \\
& =\eta_{0}\left(e^{-\frac{1}{\sqrt{U}} \delta \phi \frac{1}{\sqrt{U}}} \frac{1}{\sqrt{U}} \mathcal{Q}\left[\sqrt{U} e^{\frac{1}{\sqrt{U}} \delta \phi \frac{1}{\sqrt{U}} \sqrt{U}}\right] \frac{1}{\sqrt{U}}\right) \\
& =\eta_{0}\left(e^{-\frac{1}{\sqrt{U}} \delta \phi \frac{1}{\sqrt{U}}} Q_{B}\left[e^{\frac{1}{\sqrt{U}} \delta \phi \frac{1}{\sqrt{U}}}\right]\right)+\eta_{0}\left(\left[Q_{B} \sqrt{U}-\sqrt{U} \Psi_{R}\right] \frac{1}{\sqrt{U}}\right) \\
& \quad+\eta_{0}\left(e^{-\frac{1}{\sqrt{U}} \delta \phi \frac{1}{\sqrt{U}}}\left[\frac{1}{\sqrt{U}} Q_{B} \sqrt{U}+\frac{1}{\sqrt{U}} \Psi_{L} \sqrt{U}\right] e^{\frac{1}{\sqrt{U}} \delta \phi \frac{1}{\sqrt{U}}}\right) .
\end{aligned}
$$

The second term on the right-hand side vanishes. Note that the real solution of bosonic string field theory in (2.26) appeared in the last line of (5.9). We use the relation (4.4) applied to the real bosonic and superstring solutions shown in (4.24) and find

$$
\frac{1}{\sqrt{U}} Q_{B} \sqrt{U}+\frac{1}{\sqrt{U}} \Psi_{L} \sqrt{U}=e^{-\Phi_{0}} Q_{B} e^{\Phi_{0}},
$$

where we denoted the real superstring solution (4.19) by $\Phi_{0}$. The equation of motion can then be written as

$$
\begin{aligned}
& \eta_{0}\left(e^{-\frac{1}{\sqrt{U}} \delta \phi \frac{1}{\sqrt{U}}} Q_{B}\left[e^{\frac{1}{\sqrt{U}} \delta \phi \frac{1}{\sqrt{U}}}\right]+e^{-\frac{1}{\sqrt{U}} \delta \phi \frac{1}{\sqrt{U}}} e^{-\Phi_{0}} Q_{B}\left[e^{\Phi_{0}}\right] e^{\frac{1}{\sqrt{U}} \delta \phi \frac{1}{\sqrt{U}}}\right) \\
& =\eta_{0}\left(e^{-\frac{1}{\sqrt{U}} \delta \phi \frac{1}{\sqrt{U}}} e^{-\Phi_{0}} Q_{B}\left[e^{\Phi_{0}} e^{\frac{1}{\sqrt{U}} \delta \phi \frac{1}{\sqrt{U}}}\right]\right)=0
\end{aligned}
$$

We recognize this as the equation of motion for the original string field $\Phi$ with the following identification:

$$
e^{\Phi}=e^{\Phi_{0}} e^{\frac{1}{\sqrt{U}} \delta \Phi \frac{1}{\sqrt{U}}}
$$

This is the relation between $\delta \Phi$ and $\Phi$, which is a natural extension of (5.3) for the bosonic case.

\section{Discussion}

\subsection{Explicit construction for a class of marginal deformations}

We followed the strategy adopted in [1], and we have presented a procedure to construct solutions for general marginal deformations in the superstring from properly renormalized operator products of the marginal operator $V_{1}$ satisfying the set of assumptions listed in appendix A. In section 4 of [1], such renormalized operator products in the bosonic string were explicitly constructed for a class of marginal deformations satisfying a finiteness condition. To state it, we define operator products $\stackrel{\circ}{\circ} V_{1}\left(t_{1}\right) V_{1}\left(t_{2}\right) \ldots V_{1}\left(t_{n}\right) \circ$ for arbitrary $n$ with $t_{i} \neq t_{j}$ recursively as follows:

$$
\begin{aligned}
& { }_{\circ}^{\circ} V_{1}\left(t_{1}\right)_{\circ}^{\circ} \equiv V_{1}\left(t_{1}\right) \\
& \stackrel{\circ}{\circ} V_{1}\left(t_{1}\right) V_{1}\left(t_{2}\right) \ldots V_{1}\left(t_{n}\right) \stackrel{\circ}{\circ} \equiv V_{1}\left(t_{1}\right) \stackrel{\circ}{\circ} V_{1}\left(t_{2}\right) \ldots V_{1}\left(t_{n}\right) \stackrel{\circ}{\circ} \\
& -\sum_{i=2}^{n}\left\langle V_{1}\left(t_{1}\right) V_{1}\left(t_{i}\right)\right\rangle \stackrel{\circ}{\circ} V_{1}\left(t_{2}\right) \ldots V_{1}\left(t_{i-1}\right) V_{1}\left(t_{i+1}\right) \ldots V_{1}\left(t_{n}\right)_{\circ}^{\circ}
\end{aligned}
$$


for $n>1$ and $t_{i} \neq t_{j}$. The finiteness condition of [1] then demands that the limit

$$
\lim _{t \rightarrow t^{\prime}} \stackrel{\circ}{\circ} V_{1}(t) V_{1}\left(t^{\prime}\right)^{n \circ} \circ
$$

is finite for any positive integer $n$.

In the superstring case, we furthermore define operator products involving the operator $\widehat{V}_{1 / 2}$. For arbitrary $n$ with $t_{i} \neq t_{j}$, we define ${ }_{\circ}^{\circ} \widehat{V}_{1 / 2}\left(t_{1}\right) V_{1}\left(t_{2}\right) \ldots V_{1}\left(t_{n}\right)_{\circ}^{\circ}$ by

$$
\stackrel{\circ}{\circ} \widehat{V}_{1 / 2}\left(t_{1}\right) V_{1}\left(t_{2}\right) \ldots V_{1}\left(t_{n}\right)_{\circ}^{\circ} \equiv \widehat{V}_{1 / 2}\left(t_{1}\right) \stackrel{\circ}{\circ} V_{1}\left(t_{2}\right) \ldots V_{1}\left(t_{n}\right)_{\circ}^{\circ}
$$

and $\circ_{\circ} \widehat{V}_{1 / 2}\left(t_{1}\right) \widehat{V}_{1 / 2}\left(t_{2}\right) V_{1}\left(t_{3}\right) \ldots V_{1}\left(t_{n}\right)_{\circ}^{\circ}$ by

$$
\begin{aligned}
\circ \widehat{V}_{1 / 2}\left(t_{1}\right) \widehat{V}_{1 / 2}\left(t_{2}\right) V_{1}\left(t_{3}\right) \ldots V_{1}\left(t_{n}\right)_{\circ}^{\circ} \equiv & \widehat{V}_{1 / 2}\left(t_{1}\right) \stackrel{\circ}{\circ} \widehat{V}_{1 / 2}\left(t_{2}\right) V_{1}\left(t_{3}\right) \ldots V_{1}\left(t_{n}\right)_{\circ}^{\circ} \\
& -\left\langle\widehat{V}_{1 / 2}\left(t_{1}\right) \widehat{V}_{1 / 2}\left(t_{2}\right){ }_{\circ}^{\circ} V_{1}\left(t_{3}\right) \ldots V_{1}\left(t_{n}\right)_{\circ}^{\circ} .\right.
\end{aligned}
$$

Note that the correlation function $\left\langle\widehat{V}_{1 / 2}(t) V_{1}\left(t^{\prime}\right)\right\rangle$ vanishes because the conformal dimensions of the operators do not match so that it does not appear in the definitions (6.3) and (6.4). Then the bosonic finiteness condition (6.2) can be generalized to the following superstring finiteness conditions.

\section{The superstring finiteness conditions. The operators}

$$
\lim _{t \rightarrow t^{\prime}} \circ V_{1}(t) V_{1}\left(t^{\prime}\right)^{n \circ} \circ, \quad \lim _{t \rightarrow t^{\prime}} \circ \widehat{V}_{1 / 2}(t) V_{1}\left(t^{\prime}\right)^{n \circ} \circ
$$

are finite for any positive integer $n$ and the operator

$$
\lim _{t \rightarrow t^{\prime}} \circ \widehat{V}_{1 / 2}(t) \widehat{V}_{1 / 2}\left(t^{\prime}\right) V_{1}\left(t^{\prime}\right)^{n} \circ
$$

vanishes for any non-negative integer $n$.

We now construct explicit solutions of superstring field theory for the class of marginal deformations satisfying the superstring finiteness conditions. The operators $\left[e^{\lambda V(a, b)}\right]_{r},\left[V_{1}(a) e^{\lambda V(a, b)}\right]_{r}$, and $\left[e^{\lambda V(a, b)} V_{1}(b)\right]_{r}$ were explicitly constructed in $\S 4.3$ of [1]. When the superstring finiteness conditions are satisfied, we have

$$
Q_{B} \cdot\left[e^{\lambda V(a, b)}\right]_{r}=\left[e^{\lambda V(a, b)} O_{R}(b)\right]_{r}-\left[O_{L}(a) e^{\lambda V(a, b)}\right]_{r}
$$

with 4

$$
\begin{aligned}
& {\left[O_{L}(a) e^{\lambda V(a, b)}\right]_{r}=\lambda c(a)\left[V_{1}(a) e^{\lambda V(a, b)}\right]_{r}+\lambda \eta e^{\phi} \widehat{V}_{1 / 2}(a)\left[e^{\lambda V(a, b)}\right]_{r}-\frac{\lambda^{2}}{2} \partial c(a)\left[e^{\lambda V(a, b)}\right]_{r},} \\
& {\left[e^{\lambda V(a, b)} O_{R}(b)\right]_{r}=\lambda\left[e^{\lambda V(a, b)} V_{1}(b)\right]_{r} c(b)+\lambda\left[e^{\lambda V(a, b)}\right]_{r} \eta e^{\phi} \widehat{V}_{1 / 2}(b)+\frac{\lambda^{2}}{2}\left[e^{\lambda V(a, b)}\right]_{r} \partial c(b) .}
\end{aligned}
$$

\footnotetext{
${ }^{4}$ When the double-pole term $1 / t^{2}$ in the operator product expansion of $V_{1}(t) V_{1}(0)$ is nonvanishing, we normalize $V_{1}(t)$ such that the coefficient of the double-pole term is unity. If this convention conflicts with the reality condition on the string field, we set $\lambda=i \tilde{\lambda}$ and take $\tilde{\lambda}$ to be real when constructing the real solution.
} 
It then follows that

$$
\begin{aligned}
& {\left[\widehat{O}_{L}(a) e^{\lambda V(a, b)}\right]_{r}=\lambda c \xi e^{-\phi} \widehat{V}_{1 / 2}(a)\left[e^{\lambda V(a, b)}\right]_{r}+\frac{\lambda^{2}}{2} c \partial c \xi \partial \xi e^{-2 \phi}(a)\left[e^{\lambda V(a, b)}\right]_{r},} \\
& {\left[e^{\lambda V(a, b)} \widehat{O}_{R}(b)\right]_{r}=\lambda\left[e^{\lambda V(a, b)}\right]_{r} c \xi e^{-\phi} \widehat{V}_{1 / 2}(b)-\frac{\lambda^{2}}{2}\left[e^{\lambda V(a, b)}\right]_{r} c \partial c \xi \partial \xi e^{-2 \phi}(b) .}
\end{aligned}
$$

We can explicitly construct superstring solutions from these operators. By generalizing the calculation in appendix B.2 of [1], we can show that

$$
Q_{B} \cdot\left[O_{L}(a) e^{\lambda V(a, b)}\right]_{r}=-\left[O_{L}(a) e^{\lambda V(a, b)} O_{R}(b)\right]_{r}
$$

with

$$
\begin{aligned}
{\left[O_{L}(a) e^{\lambda V(a, b)} O_{L}(b)\right]_{r}=} & \lambda^{2} c(a)\left[V_{1}(a) e^{\lambda V(a, b)} V_{1}(b)\right]_{r} c(b)+\lambda^{2} \eta e^{\phi} \widehat{V}_{1 / 2}(a)\left[e^{\lambda V(a, b)} V_{1}(b)\right]_{r} c(b) \\
& -\frac{\lambda^{3}}{2} \partial c(a)\left[e^{\lambda V(a, b)} V_{1}(b)\right]_{r} c(b)+\lambda^{2} c(a)\left[V_{1}(a) e^{\lambda V(a, b)}\right]_{r} \eta e^{\phi} \widehat{V}_{1 / 2}(b) \\
& +\lambda^{2} \eta e^{\phi} \widehat{V}_{1 / 2}(a)\left[e^{\lambda V(a, b)}\right]_{r} \eta e^{\phi} \widehat{V}_{1 / 2}(b)-\frac{\lambda^{3}}{2} \partial c(a)\left[e^{\lambda V(a, b)}\right]_{r} \eta e^{\phi} \widehat{V}_{1 / 2}(b) \\
& +\frac{\lambda^{3}}{2} c(a)\left[V_{1}(a) e^{\lambda V(a, b)}\right]_{r} \partial c(b)+\frac{\lambda^{3}}{2} \eta e^{\phi} \widehat{V}_{1 / 2}(a)\left[e^{\lambda V(a, b)}\right]_{r} \partial c(b) \\
& -\frac{\lambda^{4}}{4} \partial c(a)\left[e^{\lambda V(a, b)}\right]_{r} \partial c(b)
\end{aligned}
$$

when the superstring finiteness conditions are satisfied. Here the operator $\left[V_{1}(a) e^{\lambda V(a, b)} V_{1}(b)\right]_{r}$ is defined as in appendix B.1 of [1]. This proves the assumption (III) stated in appendix $\mathrm{A}$. The remaining assumptions (III)-(VI) can be shown just as in [1] for the bosonic case. Thus the superstring solutions constructed from the operators $\left[\widehat{O}_{L}(a) e^{\lambda V(a, b)}\right]_{r}$ and $\left[e^{\lambda V(a, b)} \widehat{O}_{R}(b)\right]_{r}$ given in (6.9) using $\left[e^{\lambda V(a, b)}\right]_{r}$ defined in section 4 of [1] satisfy the equation of motion.

The simplest example of a deformation satisfying the superstring finiteness conditions is the deformation associated with the constant mode of the gauge field on a D-brane in flat space. If we denote a space-like coordinate along the D-brane by $X^{\mu}$ and its fermionic partner by $\psi^{\mu}$, the superconformal primary field $\widehat{V}_{1 / 2}$ associated with the marginal deformation is given by $\psi^{\mu}$, and $V_{1}$ is

$$
V_{1}(t)=G_{-1 / 2} \cdot \psi^{\mu}(t)=\frac{i}{\sqrt{2 \alpha^{\prime}}} \partial_{t} X^{\mu}(t)
$$

as in the bosonic case. In this example, the operator products (6.1), (6.3), and (6.4) are those defined by the standard normal ordering and the superstring finiteness conditions are satisfied. In $\S 4.2$ of [1], several examples of marginal deformations satisfying the finiteness condition in the bosonic case were presented. It is easy to see by generalizing the argument in $\S 4.2$ of [1] that the superstring finiteness conditions are satisfied for the supersymmetric extensions of these examples, which include deformations of flat D-branes in flat backgrounds by constant massless modes of the gauge field and of the scalar fields on the D-branes, the cosine potential for a space-like coordinate, and the hyperbolic cosine potential for the time-like coordinate. Therefore, we have explicitly constructed superstring solutions for these marginal deformations. 


\subsection{More specific assumptions}

A point where the boundary condition is changed behaves as a primary field in the bosonic case and as a superconformal primary field in the superstring case and is often described in terms of a boundarycondition changing operator. If we assume this property, we can derive more specific forms of the operators $\left[O_{L}(a) e^{\lambda V(a, b)}\right]_{r}$ and $\left[e^{\lambda V(a, b)} O_{R}(b)\right]_{r}$ both in the bosonic and superstring cases.

In the bosonic case, the BRST transformation of a primary field $V_{h}(t)$ of dimension $h$ is

$$
Q_{B} \cdot V_{h}(t)=c \partial_{t} V_{h}(t)+h(\partial c) V_{h}(t) .
$$

We thus expect that

$$
\begin{aligned}
& {\left[e^{\lambda V(a, b)} O_{R}(b)\right]_{r}=c(b) \partial_{b}\left[e^{\lambda V(a, b)}\right]_{r}+h(\lambda) \partial c(b)\left[e^{\lambda V(a, b)}\right]_{r},} \\
& {\left[O_{L}(a) e^{\lambda V(a, b)}\right]_{r}=-c(a) \partial_{a}\left[e^{\lambda V(a, b)}\right]_{r}-h(\lambda) \partial c(a)\left[e^{\lambda V(a, b)}\right]_{r},}
\end{aligned}
$$

where $h(\lambda)$ is a function of $\lambda$, which can be interpreted as the conformal dimension of the boundarycondition changing operator. Therefore, once the operator $\left[e^{\lambda V(a, b)}\right]_{r}$ for arbitrary $a$ and $b$ is given, the solution is determined up to one unknown function $h(\lambda)$. The assumption (III) in appendix $\mathrm{A}$ can now be derived from (6.14). We have

$$
\begin{aligned}
Q_{B} \cdot\left[O_{L}(a) e^{\lambda V(a, b)}\right]_{r}= & -\lim _{\epsilon \rightarrow 0} Q_{B} \cdot\left(c(a-\epsilon) \partial_{a}+h(\lambda) \partial c(a-\epsilon)\right)\left[e^{\lambda V(a, b)}\right]_{r} \\
= & -\left(c \partial c(a) \partial_{a}+h(\lambda) c \partial^{2} c\right)\left[e^{\lambda V(a, b)}\right]_{r} \\
& +\left(c(a) \partial_{a}+h(\lambda) \partial c(a)\right)\left(c(a) \partial_{a}+h(\lambda) \partial c(a)\right)\left[e^{\lambda V(a, b)}\right]_{r} \\
& +\left(c(a) \partial_{a}+h(\lambda) \partial c(a)\right)\left(c(b) \partial_{b}+h(\lambda) \partial c(b)\right)\left[e^{\lambda V(a, b)}\right]_{r} .
\end{aligned}
$$

The second line on the right-hand side precisely cancels the first line. We find

$$
\begin{aligned}
Q_{B} \cdot\left[O_{L}(a) e^{\lambda V(a, b)}\right]_{r} & =\left(c(a) \partial_{a}+h(\lambda) \partial c(a)\right)\left(c(b) \partial_{b}+h(\lambda) \partial c(b)\right)\left[e^{\lambda V(a, b)}\right]_{r} \\
& =-\left[O_{L}(a) e^{\lambda V(a, b)} O_{R}(b)\right]_{r},
\end{aligned}
$$

and thus we have derived the assumption (II). The assumptions (III) (V) in appendix $\mathrm{A}$ with additional operator insertions can also be derived from those without operator insertions.

If the conformal dimension of the boundary-condition changing operator corresponding to a deformed background is known and the function $h(\lambda)$ is determined from the BRST transformation of $\left[e^{\lambda V(a, b)}\right]_{r}$, we can identify the value of $\lambda$ which describes the deformed background. As we discussed in $\S$ 6.1, renormalized operators satisfying the assumptions of appendix A were constructed in [1] for a specific class of marginal deformations, and the function $h(\lambda)$ was determined as $h(\lambda)=\lambda^{2} / 2$ for this class of deformations. The simplest example in this class is the deformation associated with the zero mode of the gauge field (6.12), and in this case the boundary-condition changing operators at the end points $a$ and $b$ are given by $: e^{-\frac{i \lambda}{\sqrt{2 \alpha^{\prime}}} X^{\mu}(a)}:$ and $: e^{\frac{i \lambda}{\sqrt{2 \alpha^{\prime}}} X^{\mu}(b)}:$, respectively. They are primary fields of dimension $\lambda^{2} / 2$, and this is consistent with the general result $h(\lambda)=\lambda^{2} / 2$ for the class 
of deformations. The deformation by the cosine potential [51, 52, 53, 54] which interpolates Neumann and Dirichlet boundary conditions is also included in the class, and the conformal dimension of the boundary-condition changing operator between Neumann and Dirichlet boundary conditions is known to be $1 / 16$. Thus a natural conjecture is that a periodic array of lower-dimensional D-branes is described by the solution presented in section 4 of [1] with $\lambda=1 /(2 \sqrt{2})$ [

If the renormalized operator $\left[e^{\lambda V(a, b)}\right]_{r}$ obeys the relations

$$
\begin{aligned}
& \partial_{a}\left[e^{\lambda V(a, b)}\right]_{r}=\left[\partial_{a} e^{\lambda V(a, b)}\right]_{r}=-\lambda\left[V_{1}(a) e^{\lambda V(a, b)}\right]_{r}, \\
& \partial_{b}\left[e^{\lambda V(a, b)}\right]_{r}=\left[\partial_{b} e^{\lambda V(a, b)}\right]_{r}=\lambda\left[e^{\lambda V(a, b)} V_{1}(b)\right]_{r},
\end{aligned}
$$

then (6.14) can also be expressed as

$$
\begin{aligned}
& {\left[e^{\lambda V(a, b)} O_{R}(b)\right]_{r}=\left[e^{\lambda V(a, b)}\left(\lambda c V_{1}(b)+h(\lambda) \partial c(b)\right)\right]_{r},} \\
& {\left[O_{L}(a) e^{\lambda V(a, b)}\right]_{r}=\left[\left(\lambda c V_{1}(a)-h(\lambda) \partial c(a)\right) e^{\lambda V(a, b)}\right]_{r} .}
\end{aligned}
$$

It is easy to verify that the renormalized operators constructed in section 4 of [1] satisfy (6.17).

In the superstring case, the BRST transformation of a superconformal primary field $\widehat{V}_{h}(t)$ of dimension $h$ is

$$
Q_{B} \cdot \widehat{V}_{h}(t)=c \partial_{t} \widehat{V}_{h}(t)+h(\partial c) \widehat{V}_{h}(t)+\eta e^{\phi} G_{-1 / 2} \cdot \widehat{V}_{h}(t)
$$

Since

$$
\lim _{\epsilon \rightarrow 0} R(t-\epsilon) c(t)=0, \quad \lim _{\epsilon \rightarrow 0} R(t-\epsilon) \partial c(t)=-c \partial c \xi \partial \xi e^{-2 \phi}(t), \quad \lim _{\epsilon \rightarrow 0} R(t-\epsilon) \eta e^{\phi}(t)=c \xi e^{-\phi}(t),
$$

the ghost sectors of the operators $\left[\widehat{O}_{L}(a) e^{\lambda V(a, b)}\right]_{r}$ and $\left[e^{\lambda V(a, b)} \widehat{O}_{R}(b)\right]_{r}$ and consequently of the superstring solutions are highly constrained and written in terms of $c \xi e^{-\phi}$ and $c \partial c \xi \partial \xi e^{-2 \phi}$. We can again read off the unknown function $h(\lambda)$ from the BRST transformation of $\left[e^{\lambda V(a, b)}\right]_{r}$ and use it to identify the value of $\lambda$ which describes a deformed background when the conformal dimension of the corresponding boundary-condition changing operator is known.

\subsection{Pure-gauge forms}

As was discussed in appendix $\mathrm{C}$ of [1], the bosonic solutions in section 2 can be formally written as pure-gauge string fields if we use boundary-condition changing operators expanded in $\lambda$. Similarly, the superstring solutions in this paper can also be formally written as pure-gauge string fields of superstring field theory. Let us write the operator $\left[e^{\lambda V(a, b)}\right]_{r}$ as

$$
\left[e^{\lambda V(a, b)}\right]_{r}=\sigma_{L}(a) \sigma_{R}(b)
$$

\footnotetext{
${ }^{5}$ The solution, however, is not directly constructed from $\left[e^{\lambda V(a, b)}\right]_{r}$ but from its expansion in $\lambda$ with different values of $a$ and $b$ for different terms in the expansion. Furthermore, the radius of convergence in $\lambda$ of this solution is not known, so there could be possible loopholes in our argument.
} 
where $\sigma_{L}(a)$ and $\sigma_{R}(b)$ are the boundary-condition changing operators, and expand them as follows:

$$
\sigma_{L}(a)=1+\sum_{n=1}^{\infty} \lambda^{n} \sigma_{L}^{(n)}(a), \quad \sigma_{R}(b)=1+\sum_{n=1}^{\infty} \lambda^{n} \sigma_{R}^{(n)}(b) .
$$

These are formal expansions and we do not expect the operators $\sigma_{L}^{(n)}$ and $\sigma_{R}^{(n)}$ to belong to the complete set of local operators of the boundary CFT. Then the state $U$ can be formally factorized as follows:

$$
U=\Lambda_{L} \Lambda_{R}
$$

where

$$
\Lambda_{L}=1+\sum_{n=1}^{\infty} \lambda^{n} \Lambda_{L}^{(n)}, \quad \Lambda_{R}=1+\sum_{n=1}^{\infty} \lambda^{n} \Lambda_{R}^{(n)}
$$

with

$$
\left\langle\varphi, \Lambda_{L}^{(n)}\right\rangle=\left\langle f \circ \varphi(0) \sigma_{L}^{(n)}(1)\right\rangle_{\mathcal{W}_{n}}, \quad\left\langle\varphi, \Lambda_{R}^{(n)}\right\rangle=\left\langle f \circ \varphi(0) \sigma_{R}^{(n)}(n)\right\rangle_{\mathcal{W}_{n}}
$$

The states $A_{L}$ and $A_{R}$ can be written as

$$
A_{L}=-\left(Q_{B} \Lambda_{L}\right) \Lambda_{R}, \quad A_{R}=\Lambda_{L}\left(Q_{B} \Lambda_{R}\right)
$$

Let us introduce states $\Omega_{L}$ and $\Omega_{R}$ defined by

$$
\Omega_{L}=1+\sum_{n=1}^{\infty} \lambda^{n} \Omega_{L}^{(n)}, \quad \Omega_{R}=1+\sum_{n=1}^{\infty} \lambda^{n} \Omega_{R}^{(n)}
$$

with

$$
\left\langle\varphi, \Omega_{L}^{(n)}\right\rangle=\left\langle f \circ \varphi(0) Q_{B} \cdot\left[R \sigma_{L}^{(n)}(1)\right]\right\rangle_{\mathcal{W}_{n}}, \quad\left\langle\varphi, \Omega_{R}^{(n)}\right\rangle=\left\langle f \circ \varphi(0) Q_{B} \cdot\left[R \sigma_{R}^{(n)}(n)\right]\right\rangle_{\mathcal{W}_{n}}
$$

They are obviously annihilated by the BRST operator: $Q_{B} \Omega_{L}=0, Q_{B} \Omega_{R}=0$. Since

$$
\begin{aligned}
\lim _{\epsilon \rightarrow 0} R(a-\epsilon) Q_{B} \cdot \sigma_{L}^{(n)}(a) & =-\lim _{\epsilon \rightarrow 0} Q_{B} \cdot\left[R(a-\epsilon) \sigma_{L}^{(n)}(a)\right]+\sigma_{L}^{(n)}(a) \\
& =-Q_{B} \cdot\left[R \sigma_{L}^{(n)}(a)\right]+\sigma_{L}^{(n)}(a)
\end{aligned}
$$

the state $\widehat{A}_{L}$ can be written as

$$
\widehat{A}_{L}=\Omega_{L} \Lambda_{R}-U
$$

Similarly, we have

$$
\widehat{A}_{R}=U-\Lambda_{L} \Omega_{R}
$$

Thus the solutions $e^{\Phi_{L}}$ and $e^{-\Phi_{R}}$ can be written as

$$
\begin{aligned}
e^{\Phi_{L}} & =1+\left(\Omega_{L} \Lambda_{R}-U\right) U^{-1}=\Omega_{L} \Lambda_{L}^{-1}, \\
e^{-\Phi_{R}} & =1-U^{-1}\left(U-\Lambda_{L} \Omega_{R}\right)=\Lambda_{R}^{-1} \Omega_{R} .
\end{aligned}
$$


The left factor $\Omega_{L}$ of $e^{\Phi_{L}}$ and the right factor $\Omega_{R}$ of $e^{-\Phi_{R}}$ are annihilated by $Q_{B}$. Furthermore, the right factor $\Lambda_{L}^{-1}$ of $e^{\Phi_{L}}$ and the left factor $\Lambda_{R}^{-1}$ of $e^{-\Phi_{R}}$ are annihilated by $\eta_{0}$ so that both $\Phi_{L}$ and $\Phi_{R}$ are formally written as pure-gauge string fields of superstring field theory. For the particular marginal deformation (6.12) associated with turning on the zero mode of the gauge field, $\Phi_{L}$ corresponds to the solution constructed in [28].

We can also express the real solution $\Phi$ in (4.19) in terms of $\Lambda_{L}, \Lambda_{R}, \Omega_{L}$, and $\Omega_{R}$. Since

$$
e^{\Phi_{L}} U e^{-\Phi_{R}}=\Omega_{L} \Omega_{R}
$$

we have

$$
e^{\Phi}=\left(\sqrt{e^{\Phi_{L}} U e^{-\Phi_{R}}}\right)^{-1}\left(e^{\Phi_{L}} \sqrt{U}\right)=\left[\left(\sqrt{\Omega_{L} \Omega_{R}}\right)^{-1} \Omega_{L}\right]\left[\Lambda_{L}^{-1} \sqrt{\Lambda_{L} \Lambda_{R}}\right] .
$$

This expression for $e^{\Phi}$ is again formally in a pure-gauge form because the left factor is annihilated by $Q_{B}$ and the right factor is annihilated by $\eta_{0}$. Thus we have also solved the problem of finding a real superstring solution in a pure-gauge form raised in [28].

\section{Acknowledgments}

We would like to thank Volker Schomerus and Barton Zwiebach for useful discussions. The work of M.K. is supported in part by the U.S. DOE grant DE-FG02-05ER41360 and by an MIT Presidential Fellowship.

\section{A Assumptions}

In this appendix we present a list of the assumptions introduced in [1] on the renormalized operator $\left[e^{\lambda V(a, b)}\right]_{r}$ for constructing solutions corresponding to general marginal deformations. See $\S 1.1$ of [1] for more detailed discussion. While the discussion in [1] was for the bosonic string, it can be extended to the superstring if the marginal operator $V_{1}$ is the supersymmetry transformation of a superconformal primary field $\widehat{V}_{1 / 2}$ of dimension $1 / 2$ as stated in (3.1) and if the BRST operator (3.4) for the superstring is used. We believe that all the assumptions are satisfied for any exactly marginal deformation preserving superconformal invariance.

1. The BRST transformation of the operator $\left[e^{\lambda V(a, b)}\right]_{r}$ takes the following form:

$$
Q_{B} \cdot\left[e^{\lambda V(a, b)}\right]_{r}=\left[e^{\lambda V(a, b)} O_{R}(b)\right]_{r}-\left[O_{L}(a) e^{\lambda V(a, b)}\right]_{r}
$$

where $O_{L}(a)$ and $O_{R}(b)$ are some local operators at a and $b$, respectively. 
2. The BRST transformation of the operator $\left[O_{L}(a) e^{\lambda V(a, b)}\right]_{r}$ is given by

$$
Q_{B} \cdot\left[O_{L}(a) e^{\lambda V(a, b)}\right]_{r}=-\left[O_{L}(a) e^{\lambda V(a, b)} O_{R}(b)\right]_{r}
$$

The operator $\left[e^{\lambda V(a, b)}\right]_{r}$ generalizes to

$$
\left[\prod_{i=1}^{n} e^{\lambda_{i} V\left(a_{i}, a_{i+1}\right)}\right]_{r}
$$

with $a_{i}<a_{i+1}$ for $i=1,2, \ldots, n$ when different boundary conditions on different segments on the boundary are introduced. Two assumptions on this operator were made in [1].

3. Replacement. When $\lambda_{i+1}=\lambda_{i}$, the product $e^{\lambda_{i} V\left(a_{i}, a_{i+1}\right)} e^{\lambda_{i+1} V\left(a_{i+1}, a_{i+2}\right)}$ inside the operator (A.1) can be replaced by $e^{\lambda_{i} V\left(a_{i}, a_{i+2}\right)}$ :

$$
\left[\ldots e^{\lambda_{i} V\left(a_{i}, a_{i+1}\right)} e^{\lambda_{i} V\left(a_{i+1}, a_{i+2}\right)} \ldots\right]_{r}=\left[\ldots e^{\lambda_{i} V\left(a_{i}, a_{i+2}\right)} \ldots\right]_{r}
$$

4. Factorization. When $\lambda_{j}$ vanishes, the renormalized product (A.1) factorizes as follows:

$$
\left[\ldots e^{\lambda_{j-1} V\left(a_{j-1}, a_{j}\right)} e^{\lambda_{j+1} V\left(a_{j+1}, a_{j+2}\right)} \ldots\right]_{r}=\left[\ldots e^{\lambda_{j-1} V\left(a_{j-1}, a_{j}\right)}\right]_{r}\left[e^{\lambda_{j+1} V\left(a_{j+1}, a_{j+2}\right)} \ldots\right]_{r} .
$$

It was also assumed that (III) and (IV) hold when $O_{L}\left(a_{1}\right), O_{R}\left(a_{n+1}\right)$ or both are inserted into the operator (A.1). The next assumption is for operators on the family of surfaces $\mathcal{W}_{n}$.

5. Locality. The operators $\left[e^{\lambda V(a, b)}\right]_{r}$ and $\left[O_{L}(a) e^{\lambda V(a, b)}\right]_{r}$ defined on $\mathcal{W}_{n}$ coincide with those defined on $\mathcal{W}_{m}$ with $m>n$ :

$$
\begin{aligned}
{\left[e^{\lambda V(a, b)}\right]_{r} \text { on } \mathcal{W}_{n} } & =\left[e^{\lambda V(a, b)}\right]_{r} \text { on } \mathcal{W}_{m} \\
{\left[O_{L}(a) e^{\lambda V(a, b)}\right]_{r} \text { on } \mathcal{W}_{n} } & =\left[O_{L}(a) e^{\lambda V(a, b)}\right]_{r} \text { on } \mathcal{W}_{m}
\end{aligned}
$$

Finally, $e^{\lambda V(a, b)}$ is classically invariant under the reflection where $V_{1}(t)$ is replaced by $V_{1}(a+b-t)$, and it was assumed that $\left[e^{\lambda V(a, b)}\right]_{r}$ preserves this symmetry.

6. Reflection. The operator $\left[e^{\lambda V(a, b)}\right]_{r}$ is invariant under the reflection where $V_{1}(t)$ is replaced by $V_{1}(a+b-t)$ :

$$
\left[\exp \left(\lambda \int_{a}^{b} d t V_{1}(a+b-t)\right)\right]_{r}=\left[\exp \left(\lambda \int_{a}^{b} d t V_{1}(t)\right)\right]_{r} .
$$




\section{References}

[1] M. Kiermaier and Y. Okawa, "Exact marginality in open string field theory: a general framework," arXiv:0707.4472 [hep-th].

[2] E. Witten, "Noncommutative Geometry And String Field Theory," Nucl. Phys. B 268, 253 (1986).

[3] N. Berkovits, "SuperPoincare invariant superstring field theory," Nucl. Phys. B 450, 90 (1995) [Erratumibid. B 459, 439 (1996)] arXiv:hep-th/9503099.

[4] W. Taylor and B. Zwiebach, "D-branes, tachyons, and string field theory," arXiv:hep-th/0311017

[5] A. Sen, "Tachyon dynamics in open string theory," Int. J. Mod. Phys. A 20, 5513 (2005) arXiv:hep-th/0410103.

[6] L. Rastelli, "String field theory," arXiv:hep-th/0509129.

[7] W. Taylor, "String field theory," arXiv:hep-th/0605202.

[8] M. Schnabl, "Analytic solution for tachyon condensation in open string field theory," Adv. Theor. Math. Phys. 10, 433 (2006) arXiv:hep-th/0511286].

[9] Y. Okawa, "Comments on Schnabl's analytic solution for tachyon condensation in Witten's open string field theory," JHEP 0604, 055 (2006) arXiv:hep-th/0603159].

[10] E. Fuchs and M. Kroyter, "On the validity of the solution of string field theory," JHEP 0605, 006 (2006) arXiv:hep-th/0603195.

[11] E. Fuchs and M. Kroyter, "Schnabl's $\mathcal{L}_{0}$ operator in the continuous basis," JHEP 0610, 067 (2006) arXiv:hep-th/0605254.

[12] L. Rastelli and B. Zwiebach, "Solving open string field theory with special projectors," arXiv:hep-th/0606131.

[13] I. Ellwood and M. Schnabl, "Proof of vanishing cohomology at the tachyon vacuum," JHEP 0702, 096 (2007) arXiv:hep-th/0606142.

[14] H. Fuji, S. Nakayama and H. Suzuki, "Open string amplitudes in various gauges," JHEP 0701, 011 (2007) arXiv:hep-th/0609047.

[15] E. Fuchs and M. Kroyter, "Universal regularization for string field theory," JHEP 0702, 038 (2007) arXiv:hep-th/0610298.

[16] Y. Okawa, L. Rastelli and B. Zwiebach, "Analytic solutions for tachyon condensation with general projectors," arXiv:hep-th/0611110.

[17] T. Erler, "Split string formalism and the closed string vacuum," JHEP 0705, 083 (2007) arXiv:hep-th/0611200.

[18] C. Imbimbo, "The spectrum of open string field theory at the stable tachyonic vacuum," Nucl. Phys. B 770, 155 (2007) arXiv:hep-th/0611343.

[19] T. Erler, "Split string formalism and the closed string vacuum. II," JHEP 0705, 084 (2007) arXiv:hep-th/0612050.

[20] M. Schnabl, "Comments on marginal deformations in open string field theory," arXiv:hep-th/0701248.

[21] M. Kiermaier, Y. Okawa, L. Rastelli and B. Zwiebach, "Analytic solutions for marginal deformations in open string field theory," arXiv:hep-th/0701249.

[22] T. Erler, "Marginal Solutions for the Superstring," arXiv:0704.0930 [hep-th].

[23] Y. Okawa, "Analytic solutions for marginal deformations in open superstring field theory," arXiv:0704.0936 [hep-th]. 
[24] E. Fuchs, M. Kroyter and R. Potting, "Marginal deformations in string field theory," arXiv:0704.2222 [hep-th].

[25] Y. Okawa, "Real analytic solutions for marginal deformations in open superstring field theory," arXiv:0704.3612 [hep-th].

[26] I. Ellwood, "Rolling to the tachyon vacuum in string field theory," arXiv:0705.0013 [hep-th].

[27] I. Kishimoto and Y. Michishita, "Comments on Solutions for Nonsingular Currents in Open String Field Theories," arXiv:0706.0409 [hep-th].

[28] E. Fuchs and M. Kroyter, "Marginal deformation for the photon in superstring field theory," arXiv:0706.0717 [hep-th].

[29] L. Bonora, C. Maccaferri, R. J. Scherer Santos and D. D. Tolla, "Ghost story. I. Wedge states in the oscillator formalism," arXiv:0706.1025 [hep-th].

[30] T. Erler, "Tachyon Vacuum in Cubic Superstring Field Theory," arXiv:0707.4591 [hep-th].

[31] L. Rastelli and B. Zwiebach, "The off-shell Veneziano amplitude in Schnabl gauge," arXiv:0708.2591 [hepth].

[32] A. Sen and B. Zwiebach, "Large marginal deformations in string field theory," JHEP 0010, 009 (2000) arXiv:hep-th/0007153.

[33] A. Iqbal and A. Naqvi, "On marginal deformations in superstring field theory," JHEP 0101, 040 (2001) arXiv:hep-th/0008127.

[34] T. Takahashi and S. Tanimoto, "Wilson lines and classical solutions in cubic open string field theory," Prog. Theor. Phys. 106, 863 (2001) arXiv:hep-th/0107046.

[35] J. Kluson, "Exact solutions of open bosonic string field theory," JHEP 0204, 043 (2002) arXiv:hep-th/0202045.

[36] T. Takahashi and S. Tanimoto, "Marginal and scalar solutions in cubic open string field theory," JHEP 0203, 033 (2002) arXiv:hep-th/0202133.

[37] J. Kluson, "Marginal deformations in the open bosonic string field theory for N D0-branes," Class. Quant. Grav. 20, 827 (2003) arXiv:hep-th/0203089.

[38] J. Kluson, "Exact solutions in open bosonic string field theory and marginal deformation in CFT," Int. J. Mod. Phys. A 19, 4695 (2004) arXiv:hep-th/0209255].

[39] J. Kluson, "Exact solutions in SFT and marginal deformation in BCFT," JHEP 0312, 050 (2003) arXiv:hep-th/0303199.

[40] E. Coletti, I. Sigalov and W. Taylor, "Abelian and nonabelian vector field effective actions from string field theory," JHEP 0309, 050 (2003) arXiv:hep-th/0306041].

[41] N. Berkovits and M. Schnabl, "Yang-Mills action from open superstring field theory," JHEP 0309, 022 (2003) arXiv:hep-th/0307019.

[42] A. Sen, "Energy momentum tensor and marginal deformations in open string field theory," JHEP $\mathbf{0 4 0 8 ,}$ 034 (2004) arXiv:hep-th/0403200.

[43] F. Katsumata, T. Takahashi and S. Zeze, "Marginal deformations and closed string couplings in open string field theory," JHEP 0411, 050 (2004) arXiv:hep-th/0409249].

[44] H. Yang and B. Zwiebach, "Testing closed string field theory with marginal fields," JHEP 0506, 038 (2005) arXiv:hep-th/0501142.

[45] I. Kishimoto and T. Takahashi, "Marginal deformations and classical solutions in open superstring field theory," JHEP 0511, 051 (2005) arXiv:hep-th/0506240]. 
[46] N. Jokela, M. Jarvinen, E. Keski-Vakkuri and J. Majumder, "Disk Partition Function and Oscillatory Rolling Tachyons," arXiv:0705.1916 [hep-th].

[47] M. R. Gaberdiel and B. Zwiebach, "Tensor constructions of open string theories I: Foundations," Nucl. Phys. B 505, 569 (1997) arXiv:hep-th/9705038.

[48] D. Friedan, E. J. Martinec and S. H. Shenker, "Conformal Invariance, Supersymmetry And String Theory," Nucl. Phys. B 271, 93 (1986).

[49] J. Polchinski, "String theory. Vol. 2: Superstring theory and beyond," Cambridge, UK: Univ. Pr. (1998) $531 p$

[50] N. Berkovits, Y. Okawa and B. Zwiebach, "WZW-like action for heterotic string field theory," JHEP 0411, 038 (2004) arXiv:hep-th/0409018.

[51] C. G. . Callan, I. R. Klebanov, A. W. W. Ludwig and J. M. Maldacena, "Exact solution of a boundary conformal field theory," Nucl. Phys. B 422, 417 (1994) arXiv:hep-th/9402113.

[52] J. Polchinski and L. Thorlacius, "Free fermion representation of a boundary conformal field theory," Phys. Rev. D 50, 622 (1994) arXiv:hep-th/9404008.

[53] A. Recknagel and V. Schomerus, "Boundary deformation theory and moduli spaces of D-branes," Nucl. Phys. B 545, 233 (1999) arXiv:hep-th/9811237.

[54] A. Sen, "Descent relations among bosonic D-branes," Int. J. Mod. Phys. A 14, 4061 (1999) arXiv:hep-th/9902105. 